\title{
How Can a Robot Evaluate its own Behavior? A Neural Model for Self-Assessment
}

\author{
Adrien Jauffret, Caroline Grand, Nicolas Cuperlier, Philippe Gaussier and Philippe Tarroux \\ Neurocybertic team \\ ETIS laboratory, UMR 8051 \\ 95000 Cergy Cedex, France. \\ LIMSI laboratory, CNRS(UPR3251) \\ 91403 Orsay Cedex, France. \\ adrien.jauffret@ensea.fr; caroline.grand@ensea.fr \\ cuperlier@ensea.fr; gaussier@ensea.fr; philippe.tarroux@limsi.fr
}

\begin{abstract}
Allowing a robot to autonomously navigate wide and unknown environments not only requires a set of robust strategies to cope with miscellaneous situations, but also needs mechanisms of self-assessment for guiding learning and monitoring strategies. Monitoring strategies requires feedbacks on the behavior's quality, from a given fitness system, to take correct decisions.

In this work, we focus on how violations of expectations of such fitness system can be detected. Following an incremental and bio-mimetic approach, we first present two different sensorimotor strategies our robot can use to navigate: a Place Cells based strategy and a road following strategy. Then, we present a neural architecture that may be able to evaluate both navigation strategies. This model is based on an online novelty detection algorithm using a neural predictor. This neural predictor learns contingencies between sensations and actions, giving the expected sensation based from the previous perception. Prediction error, coming from surprising events, provides a direct measure of the quality of the underlying sensorimotor contingencies involved.

We propose that this model might be a key structure toward self-assessment. We made several experiments that can account for such properties for both strategies.
\end{abstract}

\section{INTRODUCTION}

In this work, one of our goal is to provide biologically plausible models for robotic navigation. Following the concepts of bio-inspired robotic and a constructivist approach we present integrated robotic control architectures resulting from a close feedback loop between experiments on animals and robots. This leads to a better understanding of the mechanisms by which the brain processes spatial information.

In previous works, we developped a model of visual Place Cells that allows the robot to exhibit simple and robust behaviors using only few Place/Action associations [2], [3]. Since this strategy has been sucessfully tested in small environment, we met issues while trying to navigate larger and more complex ones (see Section II-A). We propose to add a second simple, efficient and biologically plausible road following strategy in order to overcome issues we met with the first one (see Section II-B). Finally, we propose a generic neural architecture able to evaluate both sensorimotor navigation strategies (see Section III). We wish this model could allow the system to regulate its strategies depending on their relevance and thus improve its autonomy.

Autonomy, in the field of robotics, is still an open and poorly defined problem, for which concepts remain to be invented. By autonomous, we mean a system able to develop its knowledge and to evaluate itself to decide whether its learning or behaviors are relevant or not according to the context. This means that not only does the robot need to code its knowledge but also the limits of its knowledge. This becomes all the more important in integrated robotic systems which have to make decisions based on observations drawn from a multitude of modalities [18]. Adding self-assessment capabilities to robots should be an interesting solution, mainly in social robotics where humans play a role in the cognitive development of the robot. It could allow the system to ask human for details about its task when needed as in the collaborative control system of Fong et al. [20]. The robot could also communicate its inability to improve its learning. Moreover, it should be able to detect problems by considering aspects of novelty in its predictions. The problem of self-assessment is then sensibly close to the class of novelty detection problems. Novelty detection is a commonly used technique to detect that an input differs in some respect from previous inputs. It is a useful ability for animals to recognize an unexpected perception that could be a potential predator or a possible victim [?]. It reduces the large amount of information received by the animal so that it can focus on unusual stimuli. A variety of novelty filters have been proposed where most of them work by learning a representation of a training set (containing only normal data), then trying to underline datas that differ significantly from this training set. In the litterature, one can find different classes of methods such as statistical outlier detection, novelty detection with supervised neural networks, techniques based on self-organising map and gated dipole methods.

The standard approach to the problem of outlier detection [22], [23] is to estimate the unknown distribution $\mu$ of a set of $n$ independant random variables in order to be able to detect that a new input $X$ does not belong to the support 
of $\mu$. In the same way, extreme value theory [24] focus on distributions of data that have abnormal values in the tails of the distribution that generates the data.

The first known adaptive novelty filter is that of Kohonen and $\mathrm{Oja}$ [25]. It proposes a pattern matching algorithm where new inputs are compared with the best-matching learned pattern, meaning that non-zero output is only seen for novel stimuli. But such mechanism is certainly not biologically plausible.

Supervised neural networks methods propose also novelty detection solutions by recognizing inputs that the classifier cannot categorize reliably. Such methods estimates kernel densities to compute novelty detection in the bayesian formalism [26], [27].

An other solution is given by gated dipole fields, first proposed by Grossberg [28], [29], then used to compare stimuli and model animal's attention to novelty [32]. But systems based on gated dipoles cannot generalise to stimuli that do not have a dipole to represent them and cannot scale with the size of the dataset.

Self-organising networks also provide solutions to detect novelty using unsupervised learning [30] and particularly the so-called Adaptive Resonance Theory (ART) [35] network that uses a fixed vigilence threshold to add new nodes whenever none of the current categories represents the data. In a sense, the process of the ART network is a form of novelty detection depending on a vigilence threshold.

Neural models of memory can also detect prediction error by learning sequences of Place Cells that provides a simple means of representing pathways through the environment [36].

More recently, a model of artificial curiosity proposed by Kaplan \& Oudeyer [37] allows an agent to focus on novel stimuli to improve its learning in challenging situations, avoiding well known and totally unknown ones.

In the following we present a navigation strategy based on our previous model of visual Place Cells and its limitation for navigating complex environments.

\section{FROM TWO SENSORIMOTOR NAVIGATION STRATEGIES}

\section{A. A model of Place Cells to perform sensorimotor naviga- tion}

In previous works, we developped a biologically plausible model of the hippocampus in order to obtain visual Place Cells (VPCs) [1] that allowed controlling mobile robots for visual navigation tasks [2], [3]. The embedded pan-tilt camera allows the capture of 15 images over a 360 degrees panorama. Gradient images convolved with a difference-ofgaussian filter allows to highlight a set of salient points in the scene. A $\log$ polar transform of local views of $16 * 16$ pixels centered on each focus point is computed in order to improve pattern recognition against small rotations and scale variations.

Local views correspond to the "what" information coded in the perirhinal cortex or in other areas of the ventral visual pathway of the rat temporal cortex [5]. The azimuth of these local views (the "where" information) is provided by the parietal cortex through the parahippocampal region. The merging of "what" and "where" information may be performed in the superficial layer of the enthorinal cortex or in the postrhinal cortex [7], [6]. A neural network learns to categorize constellations of landmarks and their azimuths in the scene (5 landmarks per images) (see Fig.1) to produce place cells. Activities of the different place cells depend on the recognition level of corresponding constellations. Robustness comes from the large number of local views extracted (75 per panorama) and the use of a competition between place cells (see [4] for more details).

A neural network learns to associate a particular PC with an action (a direction to follow in our case). This sensory-motor architecture (Per-Ac [8]) allows the system to learn robust behaviors.

The activity of the cells, even in outdoor conditions, shows a peak for the learned locations (see Fig. 2) and generalizes quite correctly over large distance ( 2 to $3 \mathrm{~m}$ inside and 20 to $30 \mathrm{~m}$ outside). These broad activities are perhaps similar to the broad place cells formed in the Entorhinal Cortex.

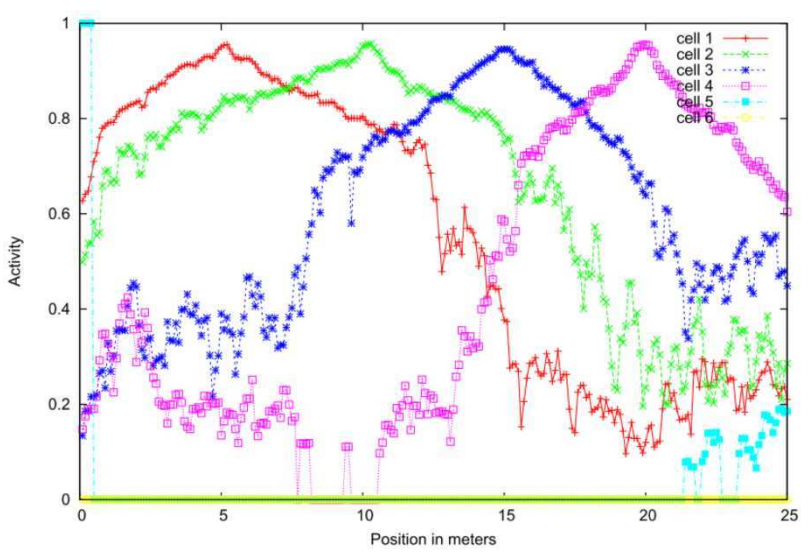

Fig. 2. Activity of 6 of our visual place cells recorded on a linear track in a real outdoor environment. The different maxima of activity correspond to the learned positions of the associated cells. Our architecture provide good generalization properties since activities present large place field. Cell 5 corresponds to a learned place outside the recording area (on the right). Cell 6 has not been recruited and shows null activity.

Even if our architecture has been succesfully tested in small sized environments (typically one room), our visualonly based mechanism shows limitations when trying to scale to larger and more complex ones (multi-room, outdoors). First the large number of Place Cells needed to cover this kind of environment introduces a computational problem that highly decreases the robustness of the localization. Moreover, we encounter some situations in outside environment where the large number of trees all around the system does not leave enough available landmarks to recognize (the entire panorama is full of green leaves that only represent noise for the system) and the only way to overcome such problem is to follow the road below. We propose to overcome these 


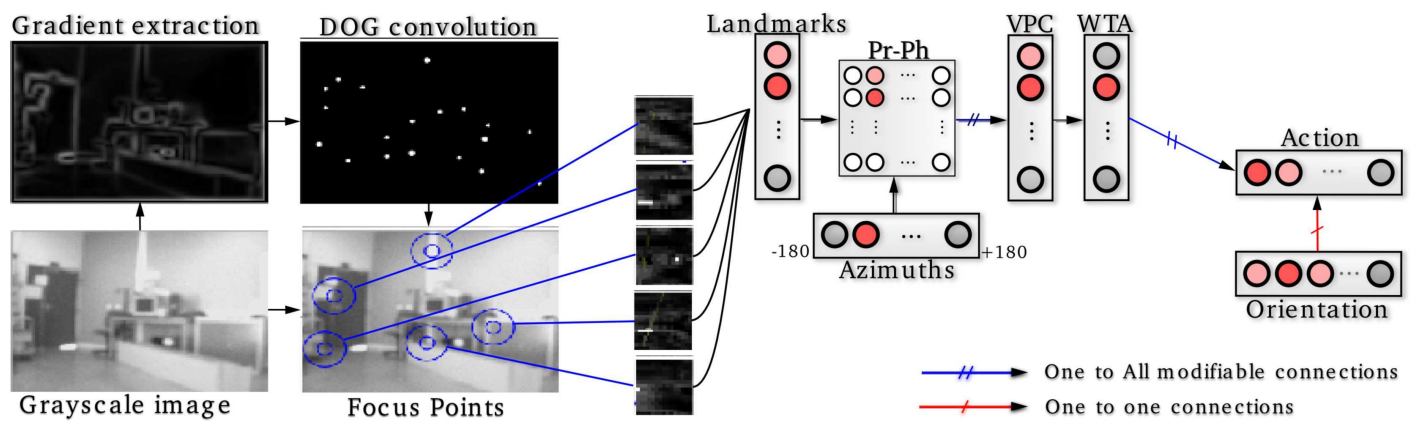

Fig. 1. Sensorimotor model relying on vision. The gradient image is convolved with a difference of gaussian filter. Local maxima of the resulting image correspond to points of interest on which the system focuses on to extract local views. A Place Cell (PC) learns to recognize a specific landmarks-azitmuths constellation. An association between the current action (robot's orientation) and this PC is learned by a least mean square algorithm (LMS), after what the system is able to move in the learned direction each time the associated PC wins.

issues by adding to our current architecture a biologically plausible road following strategy. Such strategy allows the robot to follow roads rather than learning Place Cells, in situations where it is neither necessary, nor efficient to do so. Providing two different strategies to the robot is not sufficient by itself to navigate autonomously. The system also needs a metalearning mechanism that evaluate both strategies to be able to select the right one in a given situation.

\section{B. A simple algorithm to perform road following behavior}

In this section we present a fast and robust biologically plausible road following strategy. Nowadays, several methods trying to solve this problem consist in extracting road boundaries. To detect boundaries, several methods have been proposed such as Laser-based methods [9], Radar-based [10], stereovision-based [11], color cue based [12], methods using Hough transform [13], steerable filters [14] and spline model [15]. All these methods are not suitable when the road does not exhibit significant markings or boudaries. Kong \& al. [16] propose an other algorithm which allows to find vanishing points and their confidence level using road segmentation techniques. But such method requires Gabor filters to extract textural features and road boundaries.

Here, we propose a simpler but efficient method that could be biologically plausible. Our algorithm consists in finding, among $\mathrm{N}$ (fixed number) potentially vanishing points, the one that is the best candidate. As an example, if five potentially vanishing points are proposed, there will be one in the center of the image, one on the right (resp. left) and extreme right (resp. left). Then, the robot will orient itself towards the best potential vanishing point. To find the best potential vanishing point, our method consists in determining four preferred orientations for the gradient of each pixel in the image.

The algorithm is the following one :

- acquisition of a low resolution image $(160 * 120$ pixels $)$ by a wide field camera (90 degrees field of view).

- edge extraction and gradient transformation.

- selection of the potential vanishing points: division of the image in $\mathrm{N}$ bins over the horizontal axis. Each point is placed in the center of a bin. A fixed parameter defined the skyline elevation while there is no automatic skyline detection at the moment (in following experiments, this value is set at $20 \%$ from the top of the image height).

- Four preferred directions are choosen for each vanishing point (see Fig. 3)
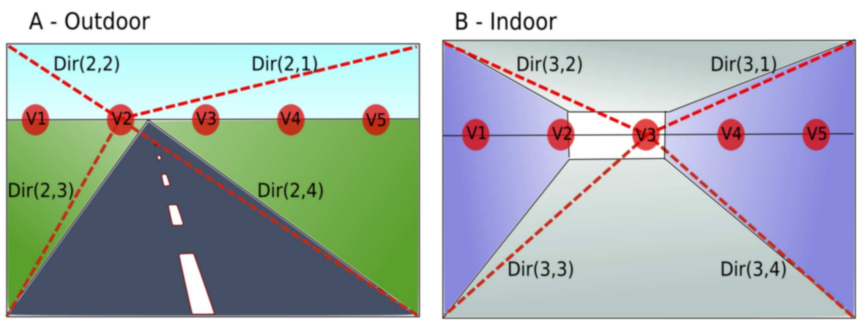

Fig. 3. Explanation of the algorithm for five potential vanishing points (respectively V1, V2, V3, V4 and V5). For outdoor and indoor cases, this figure shows how vanishing points are distributed on the image and also how the preferred direction are selected. A - For the outdoor example, the vanishing point V2 is the best candidate. B - For the indoor example, the vanishing point $\mathrm{V} 3$ is the best candidate.

- Each vanishing point $V n$ is then coded by a neuron $A C T(n)$ which activity is done by the following equations :

$$
A C T(n)=\operatorname{dir}(n, 1) \times \operatorname{dir}(n, 2)+\operatorname{dir}(n, 3) \times \operatorname{dir}(n, 4)
$$

$$
\operatorname{dir}(n, i)=\sum_{i=1}^{N} f\left(\cos \left(\theta_{v p}(i, n, l)\right) \times\left(1-\left\|\cos \left(\theta_{g}(n, l)\right)\right\|\right)\right)
$$

with :

1) $f(x)=\left\{\begin{array}{lll}x & \text { for } & 0.9<x<1 \\ 0 & \text { for } & x<0.9 \\ 1 & \text { for } & x>1\end{array}\right.$. The value of 0.9 have been choosen to allow an error of $25^{\circ}$ on each preferred direction.

2) $\theta_{v p}(i, n, l)$ corresponds to angle between a preferred direction and the vector from pixel to potential vanishing point 
3) $\theta_{g}(n, l)$ corresponds to angle between the vector from pixel to vanishing point and the vector correponding to gradient orientation

4) $\operatorname{dir}(n, 1)$ is the preferred directions top-right of neuron $n$, $\operatorname{dir}(\mathrm{n}, 2)$ top-left, $\operatorname{dir}(\mathrm{n}, 3)$ bottom-left and $\operatorname{dir}(\mathrm{n}, 4)$ bottom-right.

- Then, a simple winner takes all competition selects the best candidate between the $\mathrm{N}$ neurons.

The motor control of our model is directly inspired by control theories of Braitenberg vehicles [19]. This control is quite simple : when a vanishing point is detected on the right (resp. left), the robot will turn right (resp. left). Convergent behavior emerges from sensorimotor interactions between the system and its environment, without any need for an internal representation of the environment, or inference. Consequently, angular precision is less important than sample rate in such control.

We tested this algorithm on real images of road (See Fig.4) in several situations.
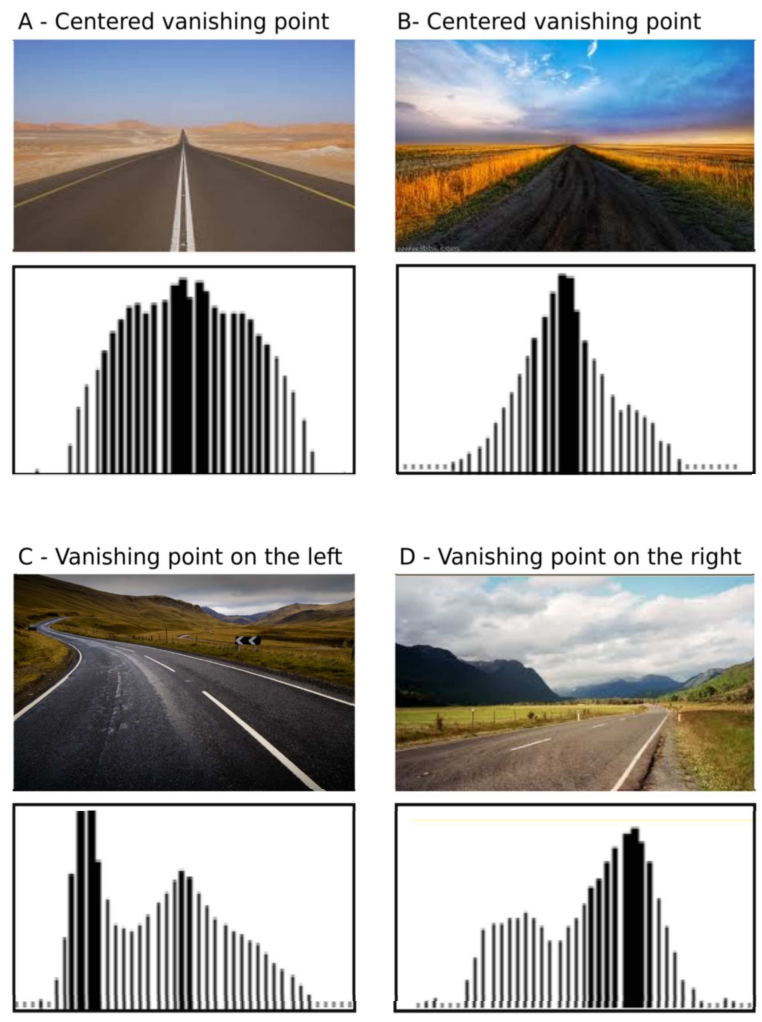

Fig. 4. Results obtained with our vanishing points detection algorithm on real images. For each cases: Up - Real image of a road. Down- Activity levels of 41 neurons, each one firing for a particular vanishing point on the image. A - In a simple case (road with boundaries), the vanishing point is well detected in the center of the image and generalized quite correctly to neighborhood $\mathbf{B}-$ Without boundaries, the vanishing point remain salient since there is a significant gradient between road and grass. $\mathbf{C}-$ For a twisting road, our algorithm detect two potential vanishing points, a centered one and the other on the left side. Nevertheless, the more active is the one on the left. D-Exemple of a vanhing point detected on the right side.

Thanks to this method, our system is able to follow any types of vanishing points such as roads, corridors, paths or railways. Furthermore, this algorithm has a satisfying framerate of 20 images per secondes (for 41 vanishing point neurons) and this framerate could increase if we consider less neurons. Such high framerate is obtained because only the higher gradients are considered in our algorithm. Therefore, the intensity of the gradient have been normalized by using the cosinus of the angle. So, in Fig.4 (case B) the gradient of road edges is not really high even though the vanishing point is detected.

A drawback of our method is the adjustement of the skyline position. Moreover, in some environments, the vanishing lines over the skyline could be an information (like in a corridor or in forest) although high relieves or clouds that can disturb localization of the vanishing point.

\section{TOWARDS A FIRST METHOD FOR SELF-MONITORING}

Here we present a generic model for self-assessment based on novelty detection techniques. Our model consists in two steps. First, to learn sensorimotor contingencies induced by the navigation strategy involved in a normal situation (training set), second to be able to detect extraneous sensorimotor patterns in novel situations.

Our model is as follow (see Fig.5) :

Lets denote $y$, a vector of $n$ neurons $y_{i}$ relative to agent's own sensations. It can be both place cells or vanishing point cells in our case. $y$ can be viewed as a set of random variable $y_{i}$.

$x$ is a vector of neurons $x_{i}$ relative to agent's proprioception, where the winning neuron code for the current orientation. $P$ is the tensorial product between $x$ and $y$ with recurrent connections of weight $\alpha$. $P$ codes a short term memory of the agent's perception, where $P_{i}, j$ denote the particular tuple of both $x_{i}$ and $y_{i}$ neurons.

Here we defined Perception as the integral of all Sensations and Actions [31]:

$$
\operatorname{Per}(t)=\int_{-\infty}^{t} \operatorname{Sen}(t) \cdot A c(t)
$$

Where Sen denotes a vector of sensations (the input vector $y$ in our case), and $A c$ denotes a vector of actions (the proprioceptive vector $x$ coding for the current orientation). In our model, the matrix $P$ estimates the robot's perception by integrating sensations and actions in a finite shifting temporal window defined by the recurrent weight $\alpha$ :

$$
\begin{gathered}
P(t+1)=\alpha \cdot P(t)+(1-\alpha) \cdot \operatorname{Sen}(t) \cdot A c(t) \\
P(t)=\sum_{i=0}^{t} \alpha^{i-1} \cdot(1-\alpha) \cdot \operatorname{Sen}(t) \cdot A c(t)
\end{gathered}
$$

Basically, it means that recent inputs have a higher weight in our process than older ones. This type of filter has been tested by Richefeu and Manzanera [17] in a motion detection context. The parameter $\alpha$ is used in order to attach more importance to the near past than to the far past. 
Then, a vector $\hat{y}$, same size as $y$, estimates the mean $E[y]$ of the current sensation $y$ from the perception matrix $P$ by a least mean square algorithm (LMS) [33]. We make the assumption that $y$ follows a Gaussian distribution required by least-squares. An absolute difference between $y$ and $\hat{y}$ represents the instant error vector $e$. In the same manner, a vector $\hat{e}$, estimates the first moment about the mean, $\mu_{2}=E[e]$, of the current error $e=y-\hat{y}$ from the perception matrix $P$ by a LMS algorithm. The second order error is defined as $e_{2}=e-\mu_{2}$. The second moment about the mean is defined as $\mu_{3}=E\left[\left|e-\mu_{2}\right|\right]$

The third order error is defined as $e_{3}=e_{2}-\mu_{3}$. Novelty $N$ is defined as the global fourth moment about the mean. $N$ is a single neuron that integrates all $e_{3}$ neurons activities: $N=\left|\sum_{i=1}^{n} e_{3 i}\right| . N$ is summarized by :

$$
\begin{aligned}
& N=E\left[\mid \sum_{i=1}^{n}\left(\left|y_{i}-E\left[y_{i}\right]\right|-E\left[\left|y_{i}-E\left[y_{i}\right]\right|\right]\right)\right. \\
& -E\left[\left|y_{i}-E\left[y_{i}\right]\right|-E\left[\left|y_{i}-E\left[y_{i}\right]\right|\right]\right]
\end{aligned}
$$

$N$ represent the prediction error of the network, that will be used to detect unexpected events. The different moments about the mean $\mu_{2}, \mu_{3}$ and $\mu_{4}$ represent respectively the pseudo-variance, the pseudo-skewness and the pseudokurtosis (while their measure follows the L1-norm rather than the L2-norm).

Our architecture is thus able to learn an internal model of the dynamical interactions the system has with the external world.

\section{EXPERIMENTS AND RESULTS.}

We have tested our model of self-evaluation in several situations for both strategies. Following experiments run in 2 different 3-dimensional simulated environments. We used a simulated robotic platform that is $40 * 40 \mathrm{~cm}$ wide and equiped with 2 wheels, proximity sensors for obstacle avoidance and a pan-tilt camera used to extract points of interest in the visual panorama.

The place/action strategy has been tested in a simulated room (Setup 1: see Fig.6) of $15 * 15 \mathrm{~m}$ with a uniform floor and salient landmarks on walls. The robot is trained by an human teacher (supervised learning) to perform a round path by learning Place Cell/Action associations. As a stereotypical human/dog training interaction, the teacher uses a leash to pull the robot in the desired direction. Thus, the robot is detecting prediction error by comparing human order to its own will. This novelty detection neuromodulates the vigilence of the system so that it decides to recruit a new place cell and learns the association to its current orientation. Following such interactions, the robot is able to learn the path the human is teaching. A proscriptive learning (correcting the system rather than showing it the path) is necessary to get a stable and robust attraction field [34]. No more than 8 place/action associations are sufficient to perform a robust round trip in our experiment. The evaluation mechanism learns the sensorimotor contingencies while the robot performs its round trip in a normal situation (similar to the training set). In this setup, the vector of sensation $y$ is defined by the vector of 8 Place Cells learned by the system. We set the recurrent weight $\alpha=0.95$ empirically, based on the frequency of changes in sensations. The sensorimotor loop of that strategy is quite slow since states only change when the robot navigates from one place to an other (it mainly depends on the distance between 2 places and the robot's linear velocity). Indeed, an $\alpha$ near to 1 results in a long temporal window (old states are more important than recent ones). 3 laps were necessary for the evaluation mechanism to completely predict its sensation from all sensorimotor situations perceived during the trip. Indeed, learning is completed only when the novelty level reaches a minimum (typically below 0.4 ) and stagnates in all places.

The road following strategy has been tested in a simulated outside environment (Setup 2: see Fig.6) of 40*40m with white roads over grass on the floor and trees texture on walls. The system is then able to correctly follow roads when one is in its field of view. On the other side, navigating on grass results in random movements since there is no stable and well-defined vanishing point to follow. The evaluation mechanism learns the sensorimotor contingencies while the robot performs road following in a normal situation (training set). In this setup, the vector of sensation $y$ is defined by 13 vanishing point neurons processed by the system. We set the recurrent weight $\alpha=0.7$ empirically, based on the velocity of the sensorimotor loop. Indeed, the sensorimotor loop of that strategy is significantly faster than for the place/action strategy while vanishing point states change at a speed that directly depends on the robot's angular velocity. 5 minutes of navigation were necessary for the system to completely predict its sensation from sensorimotor situations perceived while following a road. Learning is completed when the novelty level reaches a minimum of 0.4 and stagnates.
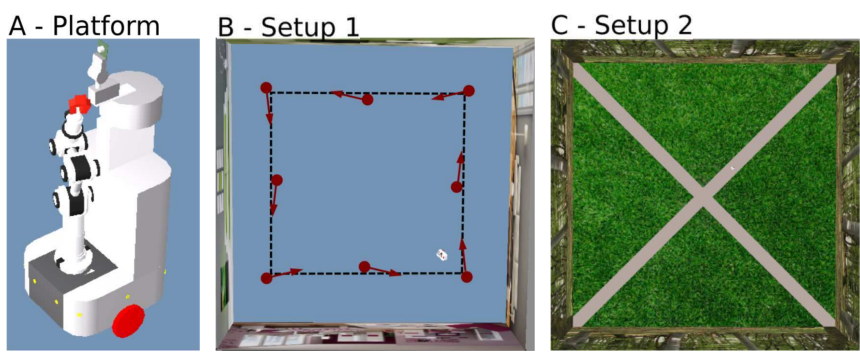

Fig. 6. Experimental setup for the different strategies. A- Robotic platform used in our simulations. $\mathbf{B}-$ Setup used to test the place/action strategy. The system evolve in a simulated room of $15^{*} 15 \mathrm{~m}$. It learns 8 places associated with different actions (in red) to perform an ideal round behavior (black dotted line). $\mathbf{C}-$ Setup used to test the road following strategy. The system evolve in a simulated outside environment of $40 * 40 \mathrm{~m}$. The robot can navigate both on roads or on grass.

\section{A. Results relying on Place/Action experiments}

After the system has completely learned the desired trajectory (Setup 1) and sensorimotor predictions relative to this trajectory, we tested it in several situations to show the 


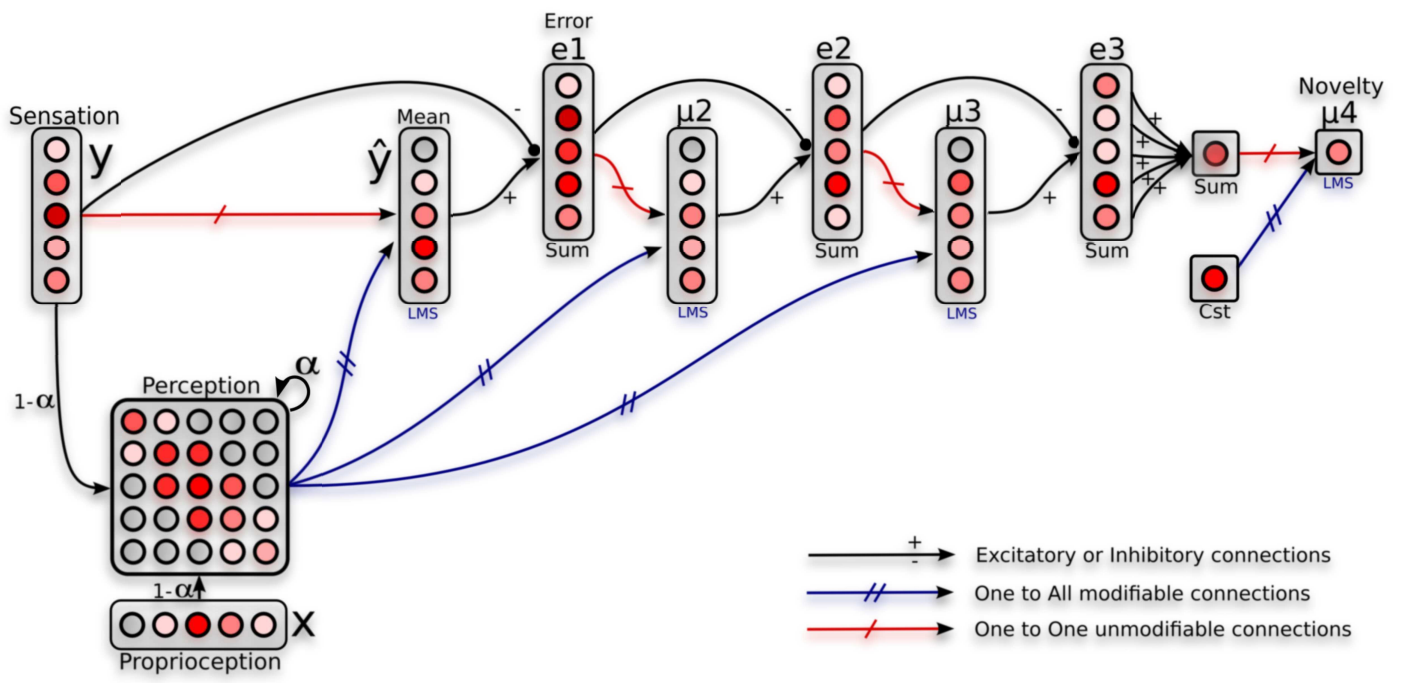

Fig. 5. Neural architecture relying on self-evaluation. A network learns the sensorimotor law of a strategy by learning to predict the current sensation from the previous perception. We defined perception as the short term memory of recent sensation/action tuples. Such perception matrix is used to predict an approximation of the 3 first moments about the mean of the sensation. A global novelty level gives to the system a direct feedback on the quality of the behavior involved.

robustness of our model to detect whether such situation is normal or abnormal. In a first experiment, we tested the robustness of the strategy in a normal situation. The robot performs 12 standard laps without perturbation. Results show a robust and stable behavior with a trajectory close to the desired one. The novelty level stays relatively low since it never gets over 0.4 , with a mean value of 0.2. It defines the minimum prediction error the system is able to learn. Such minimum error is directly linked to the degree of deepness of the prediction process. Since we defined the novelty as the fourth moment about the mean, our model is not able to characterize statistical variations over such precision. A fifth and sixth moment should be able to respectively learn the novelty mean and variance.

In a second experiment, we introduce an obstacle in the environment so that the system is forced to avoid it (direct priority is given to the obstacle avoidance strategy by a subsomption architecture). The system gets around that obstacle and successfully goes back to its original path thanks to the generalization properties place cells provide. Novelty level shows peaks when the system is avoiding the obstacle, since the orientation taken does not correspond to the learned one in that place.

In an other experiment, we propose to put off the light suddenly so that the visual system is not able to maintain coherent place cells activity. Thus, the system is not able to recognize places anymore and becomes totally lost. It results in random movements. Novelty level shows a sudden offset but keep more or less the same variance. Indeed, the system is not able to recognize places anymore, even if it tries to predict it.

In the same way, the last experiment proposes to suddenly shift the north direction by 90 degrees. The robot behavior tends to be random after a few seconds. The novelty level shows large variations after the event. Indeed, the system sometimes takes an unexpected orientation, sometimes a predicted one.

\section{B. Results relying on road following experiments}

This time the system evolves in the simulated outside environment previously described (Setup 2) and tries to perform road following. The evaluation mechanism is trained to predict the sensorimotor laws involved in following a road. In a first experiment, the environment only contains one single road stopping at the middle of it, and grass elsewhere. The robot starts on grass, in a corner, oriented towards the road. Results show that it converges into the road in order to be aligned with the road, then the system correctly follows it until its end. Finally, it ends its trip by random movements onto grass. Novelty level shows a progressive decrease while the robot converges into the road, then stays minimum and quite stable while following it. Novelty level increases progressively when leaving the road and stays high until the end of the experiment.

In a second experiment, we propose to test the robustness of the self-evaluation mechanism on a 1 hour navigation experiment. This time, the environment contains 2 crossing roads covering diagonal distances (as seen in Fig.6: Setup 2 ). The purpose of this experiment is to test the evaluation mechanism in almost all different situations. Results show that the evaluation mechanism is able to differentiate between normal and abnormal sensorimotricity quite correctly since it predicts well its sensation while navigating on both roads, and detects novelty anywhere else (on grass).

\section{Discussion}

In this incremental design approach, one objective of our robotic experiments is to show the limitations of models. Hence, since our previous works on modeling place cells 

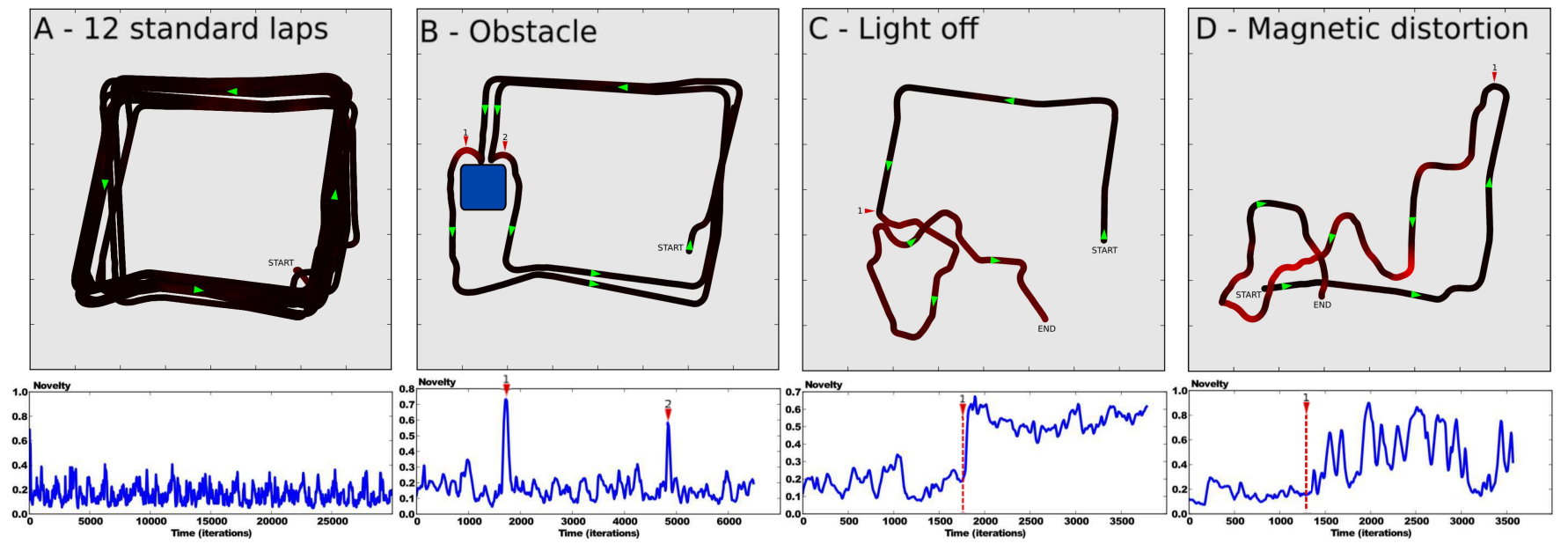

Fig. 7. Results obtained for the place/action strategy (Setup 1). A- Up : Trajectory of the robot after performing 12 laps autonomously without disturbance. Green Arrow indicate movements direction. Down : Evolution of the novelty level in time. Novelty level stays below 0.5 with a constant variance as no abnormal event appears. B- Up : 2 laps trajectory of the robot while an obstacle suddenly appears in the environment. Obstacle avoidance allows the robot to get over this situation. Generalization capabilities of the place cells allow it to go back into the learned path. Down : Novelty level shows peaks for respectively the first (1) and second (2) time the system faces the obstacle. C- Up : The system perform its task correctly (from START to 1) when the ambient light is suddenly switched off (1). It results in random movements, as no cues are visible. The robot is totally lost (from 1 to END) Down : Novelty mean level suddenly grows by an offset corresponding to the error produced by pure random predictions. $\mathbf{D}-\mathbf{U} \mathbf{p}$ : The system perform its task correctly (from START to 1) when the north orientation is suddenly shifted by 90 degrees (1). It results in random movements since unexpected actions are performed in each places (from 1 to END). Down : Novelty variance grows significantly.
A - Single test on a road

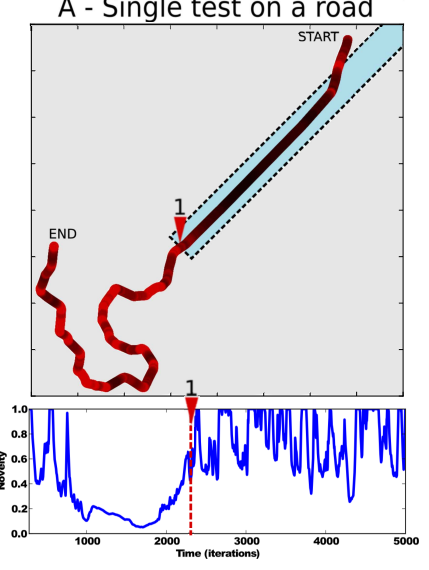

B - 1 hour of road following

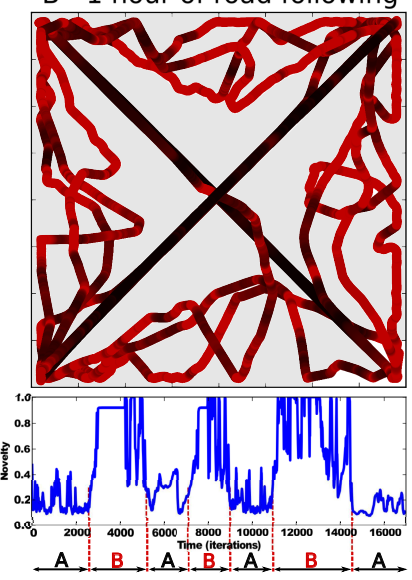

Fig. 8. Results obtained in simulated outside environments using the road following strategy (Setup 2). A- Up : Trajectory of the robot on a single test. There is only one single road (dotted line) ending in the middle of the open-space. The robot starts on grass, converge into the road, then follows it until its end. Up : Novelty level shows a decrease as the system converge into the road. It grows up progressively at the end of the road. $\mathbf{B}-\mathbf{U p}$ : Trajectory of the robot after 1 hour of autonomous navigation in a crossroad environment. Color indicates novelty level (Red:1, Black:0). Down : Evolution of the novelty level for a selected sample of time. Results show a minimum novelty level (below 0.5 ) when the system follows roads (A) and a high novelty level when navigating on grass (B) without any road to follow.

allows our system to perform simple sensorimotor behaviors in small size environments, we highlight the need to take into account solutions to overcome some issues we met while trying to navigate more complex ones. First, we extended our architecture by adding a robust and biologically plausible road following strategy. Such strategy allows the robot to naturally converge into visible roads, following it on a robust manner. It provide a second strategy to navigate in situations where learning Place Cells is neither necessary, nor efficient. Then, as using two different strategies is not sufficient by itself to navigate autonomously, we showed that the system also needs a mechanism that evaluate both strategies in order to regulate these strategies. We proposed a model for self-assessment based on statistical outlier detections in a dynamic point of view. We defined perception as an internal model of the sensorimotor interactions the system has with the external world. Such model of perception provide a generic grounding to perform predictions on agent's sensation. Novelty is then defined as the prediction error at a $n^{\prime}$ th level, depending on the desired accuracy. Results show that our model presents good generalization capabilities since the same architecture can work for at least two different sensorimotor strategies.

Current work focuses on using this self-assessment mechanism as an active judge to monitor (select or inhibit) different strategies in particular contexts. The mechanism should therefore select useful strategies, while discarding others, based on its own evaluation of a given behavior in a specific context. An emotional controller (involving basic emotions like frustration and satisfaction), will be necessary to take decisions. Moreover, the robot may stop and call for help if no relevant strategies are found (if switching strategy does not increase any progress at all). We will evaluate the model performance on long range outside experiments (navigating several kilometers) on a real outdoor robot. 


\section{ACKNOWLEDGMENT}

We would like to thank AUTO-EVAL, the NEUROBOT French ANR project. This project was also supported by the Centre National de la Recherche Scientifique (CNRS) and the Delegation Generale pour l'Armement (DGA).

\section{REFERENCES}

[1] J.OKeefe and N. Nadel. The hippocampus as a cognitive map. Clarendon Press, Oxford, 1978.

[2] P. Gaussier, A. Revel, J.-P. Banquet, and V. Babeau. From view cells and place cells to cognitive map learning: processing stages of the hippocampal system. Biological Cybernetics, 86:1528, 2002.

[3] J.-P. Banquet, P. Gaussier, M. Quoy, A. Revel, and Y. Burnod. A hierarchy of as- sociations in hippocampo-cortical systems: Cognitive maps and navigation strategies. Neural Computation, 17(6):13391384, June 2005.

[4] C. Giovannangeli, P. Gaussier, and J. P. Banquet. Robustness of visual place cells in dynamic indoor and outdoor environment. International Journal of Advanced Robotic Systems, 3(2):115124, June 2006.

[5] B. Kolb and R. Tees. The Cerebral Cortex of the Rat. MIT Press, 1990.

[6] R. D. Burwell and D. M. Hafeman. Positional ring properties of postrhinal cortex neurons. Neuroscience, 119(2):577588, 2003.

[7] W. A. Suzuki, E. K. Miller, and R. Desimone. Object and place memory in the macaque entorhinal cortex. J Neurophysiol, 78(2):10621081, Aug 1997.

[8] P. Gaussier and S. Zrehen. Perac: A neural architecture to control artificial animals. Robotics and Autonomous Systems, 16(2-4):291 320, 1995. Moving the Frontiers between Robotics and Biology.

[9] J. Sparbert, K. Dietmayer, and D. Streller. Lane detection and street type classification using laser range images. IEEE Proceedings in Intelligent transportation Systems, 2001.

[10] B. Ma, S. Lakshmanan, and A. O. Hero. Simultaneous detection of lane and pavement boundaries using model-based multisensor fusion. IEEE Transactions on Intelligent Transportation Systems, 2000.

[11] M. Bertozzi and A. Broggi. Gold: A parallel real-time stereo vision system for generic obstacle and lane detection. IEEE Transactions on Image Processing, 1998.

[12] S.-J. T. Tsung-Ying Sun and V. Chan. Hsi color model based lanemarking detection. IEEE Intelligent Transporta- tion Systems Conference, 2006.

[13] B. Yu and A. K. Jain. Lane boundary detection using a multiresolution hough transform. ICIP, 1997.

[14] W. T. Freeman and E. H. Adelson. The design and use of steerable filters. PAMI, 1991.

[15] Y. Wang, E. K. Teoh, and D. Shen. Lane detection and tracking using b-snake. Image and Vision Computing, 2004.

[16] Kong, H. and Audibert, J.Y. and Ponce, J. Vanishing point detection for road detection. Computer Vision and Pattern Recognition, 2009. CVPR 2009. IEEE Conference on, 96-103, 2009.

[17] Richefeu, Julien and Manzanera, Antoine. A new hybrid differential filter for motion detection. Computer Vision and Graphics. 2006. Springer, 727-732.

[18] Zillich, M.; Prankl, J.; Morwald, T.; Vincze, M.; , Knowing your limits - self-evaluation and prediction in object recognition, Intelligent Robots and Systems (IROS), 2011 IEEE/RSJ International Conference on, vol., no., pp.813-820, 25-30 Sept. 2011. doi: 10.1109/IROS.2011.6094856.
[19] Braitenberg, V. Vehicles: Experiments in synthetic psychology. 1986. MIT press.

[20] Terrence W. Fong, Illah Nourbakhsh, and Kerstin Dautenhahn, "A survey of socially interactive robots," Robotics and Autonomous Systems, , 2003.

[21] Stephen Marsland. Novelty detection in learning systems. Neural Computing Surveys, 3:157-195, 2003.

[22] Zbynek Sidak, Pranab K. Sen, Jaroslav Hajek. Theory of Rank Tests. 1967.

[23] L. Devroye and G. L. Wise, Detection of abnormal behavior via nonparametric estimation of the support, SIAM J. Appl. Math. 38 (1980) 480-488.

[24] Emil Julius Gumbel (1958) Statistics of Extremes, Columbia University Press.

[25] Teuvo Kohonen and E. Oja. Fast adaptive formation of orthogonalizing filters and associative memory in recurrent networks of neuron-like elements. Biological Cybernetics, 25:8595, 1976.

[26] Christopher M. Bishop. Novelty detection and neural network validation. IEEE Proceedings on Vision, Image and Signal Processing, 141(4):217222, 1994

[27] Stephen Roberts and Lionel Tarassenko. A probabilistic resource allocating network for novelty detection. Neural Computation, 6:270 284, 1994.

[28] Stephen Grossberg. A neural theory of punishment and avoidance. I. Qualitative theory. Mathematical Biosciences, 15:3967, 1972a.

[29] Stephen Grossberg. A neural theory of punishment and avoidance. II. Quantitative theory. Mathematical Biosciences, 15:253285, 1972b.

[30] S. Kaski, J. Kangas, and T. Kohonen. Bibliography of self-organising map (SOM) papers: 1981 1997. Neural Computing Surveys, 1:102 350, 1998.

[31] M. Maillard , O. Gapenne, L. Hafemeister, P. Gaussier. Perception as a dynamical sensori-motor attraction basin (2005), Advances in Artificial Life (8th European Conference, ECAL).

[32] Daniel S. Levine and Paul S. Prueitt. Simulations of conditioned perseveration and novelty preference from frontal lobe damage. In Michael L. Commons, Stephen Grossberg, and John E.R. Staddon, editors, Neural Network Models of Conditioning and Action, chapter 5, pages 123 147. Lawrence Erlbaum Associates, Hillsdale, NJ, 1992.

[33] B. Widrow and M.E. Hoff, Jr. Adaptive Switching Circuits. IRE WESCON Convention Record, 4:96-104, August 1960.

[34] C. Giovannangeli and $\mathrm{Ph}$. Gaussier. Learning to Navigate, SelfEvaluation and Progress 6th International Conference on Epigenetic Robotics: Modeling Cognitive Development in Robotic (Epirob'06) 2006

[35] Gail A. Carpenter and Stephen Grossberg. The ART of adaptive pattern recognition by a selforganising neural network. IEEE Computer, 21:77 88, 1988.

[36] Hasselmo, Michael E; McClelland, James L. Neural models of memory. Volume 9 (2) Elsevier Apr 1, 1999. 0959-4388. doi: 10.1016/S0959-4388(99)80025-7.

[37] Oudeyer P-Y, Kaplan , F. and Hafner, V. (2007) Intrinsic Motivation Systems for Autonomous Mental Development, IEEE Transactions on Evolutionary Computation, 11(2), pp. 265-286. DOI: 10.1109/TEVC.2006.890271 